INGENIERÍA MECÁNICA

\title{
Diseño, simulación, fabricación y caracterización de una turbina tipo Pelton de $5 \mathrm{~kW}$
}

MECHANIC ENGINEERING

\section{Design, simulation, construction and characterization of a 5 kW hydraulic Pelton turbine}

\author{
Sergio Agudelo*§, Edwin Chica*, Felipe Obando*, Natalia Sierra*, Laura Velásquez*, \\ Walter Enríquez* \\ * Departamento de Ingeniería Mecánica, Universidad de Antioquia, Medellín, Colombia \\ §seragude@udea.edu.co,echica@udea.edu.co,felipeobando@udea.edu.co,nataliasierra@udea.edu. \\ co,lauraisabel03@hotmail.com,walterenriquez77@hotmail.com
}

(Recibido: Noviembre 15 de 2012 - Aceptado: Abril 16 de 2013)

\begin{abstract}
Resumen
Se presenta la metodología empleada para la construcción de un microgenerador hidráulico de $5 \mathrm{~kW}$ con turbina tipo Pelton. Para unas condiciones de operación (cabeza hidráulica y caudal) y a partir de una base teóricoexperimental, se modelaron los componentes con ayuda de herramientas computacionales tipo CAD (Diseño asistido por computador), CAE (Ingeniería asistida por computador) y CFD (Análisis computacional fluidodinámico). Posteriormente, se empleó un software CAM (Manufactura asistida por computador) y procesos de fundición para la fabricación de los componentes del microgenerador. Por último, y con el fin de validar el diseño, se realizó la caracterización del microgenerador mediante pruebas en el Laboratorio del Grupo de Investigación de Energía Alternativa de la Universidad de Antioquia. A partir de las pruebas realizadas, fue posible cuantificar la eficiencia de la turbina Pelton, encontrar la relación óptima entre la apertura del inyector y la potencia generada, la velocidad óptima de operación, la eficiencia global del microgenerador y las condiciones hidráulicas en las cuales el microgenerador puede ser utilizado, brindando la mayor eficiencia de generación de energía eléctrica.
\end{abstract}

Palabras claves: $C A D, C F D$, microgenerador, turbina, pelton.

\begin{abstract}
It is presented the methodology for the construction of a $5 \mathrm{~kW}$ hydraulic microgenerator, with a Pelton turbine. For a given operating conditions (hydraulic head and flow) and from a theoretical and experimental basis, the different components were modeled using computational tools such as CAD (Computer Aided Design), CAE (Computer Aided Engineering) and CFD (Computer Fluid Dynamics). Subsequently, it was used a CAM (Computer Aided Manufactured) software and casting processes for manufacturing the components of the microgenerator. Finally, and in order to validate the design, characterization was performed by testing the microgenerator in the Laboratory of Alternative Energy Research Group at the Universidad de Antioquia. From the tests performed, it was possible to quantify the efficiency of the Pelton turbine, to find the optimum ratio between the opening of the nozzle and the generated power, the optimum speed of operation, the overall efficiency of the microgenerator and the hydraulic conditions in which the microgenerator can be used providing an optimal efficiency of electric power generation.
\end{abstract}

Keywords: $C A D, C F D$, microgenerator, pelton, turbine. 


\section{Introducción}

La microgeneración hidroeléctrica se emplea como solución energética en zonas que carecen del servicio de energía eléctrica; sin embargo, poco se conoce sobre experiencias de diseño y fabricación de microgeneradores hidráulicos para el aprovechamiento de un recurso hídrico específico. Por lo general, se emplean microgeneradores que han funcionado adecuadamente en un rango de valores de cabeza y caudal determinados, pero se desconoce su eficiencia de acuerdo a las condiciones de operación a las cuales está siendo sometido (ITDG, n.d).

Con el fin de dar solución a este problema, se desarrolla una metodología para diseñar y construir microgeneradores hidráulicos con turbina tipo Pelton para condiciones de operación de caudal (Q) y cabeza $(\mathrm{H})$ específicos, empleando herramientas computacionales $(\mathrm{CAD}$, CAE, CFD), materiales y procesos de fabricación de vanguardia que permitan la mayor eficiencia en el aprovechamiento energético del recurso hidráulico. Los microgeneradores se diseñaron asegurando que posean un adecuado rendimiento en la conversión de energía para disminuir los tiempos de amortización de los equipos y el costo del $\mathrm{kW}$-h producido.

\section{Metodología de diseño}

\subsection{Modelación}

Para el diseño del microgenerador, se empleó la metodología presentada en el diagrama de flujo de la figura 1 Las variables de entrada para el diseño son las condiciones hidráulicas del sitio final de instalación (caudal y cabeza), la potencia eléctrica que se desea generar, la velocidad de giro de la turbina y la eficiencia global de generación de energía.

Al definir la eficiencia global de generación, la velocidad de giro de la turbina y la potencia eléctrica requerida es posible definir una relación entre los valores de caudal y cabeza que pueden proporcionar la potencia requerida, cumpliendo con las recomendaciones del rango de velocidad específica reportadas en la literatura para este tipo de máquinas.

La potencia hidráulica aprovechada por la turbina está dada por la relación entre las características $(\mathrm{Q}, \mathrm{H})$ del sitio donde se utiliza el microgenerador. Además, está en función de la rugosidad superficial del álabe; de la velocidad de rotación de la turbina; del ángulo y de la velocidad de entrada y de salida del agua al álabe. Debido a que el chorro de agua está muy cerca del álabe, se supone que la magnitud de la velocidad de salida del chorro es la misma que la velocidad de entrada del agua al álabe. Todos estos parámetros se relacionan entre sí mediante el triángulo de velocidades mostrado en la figura 2, donde: $\alpha_{2}$ es el ángulo absoluto con el cual el agua sale del álabe, $\beta_{2}$ es el ángulo de salida del álabe, $\mathrm{U}_{1}$ y $\mathrm{U}_{2}$ son las velocidades tangenciales, $\mathrm{W}_{1} \mathrm{y} \mathrm{W}_{2}$ son las velocidades relativas y $\mathrm{C}_{1} \mathrm{y}_{2}$ son las velocidades absolutas del chorro de agua a la entrada y a la salida del álabe, respectivamente.

Conociendo la velocidad de salida del chorro y el caudal aplicado, se calcula el diámetro nominal que debe tener el chorro. Las dimensiones del inyector dependen de este valor. Se debe garantizar que la variación de la posición de la aguja reguladora permita disminuir y aumentar el diámetro del chorro alrededor del valor nominal calculado con el fin de variar la potencia hidráulica aplicada a la turbina.

Ya que el diámetro nominal del chorro está relacionado con la potencia aprovechada por la turbina, se utiliza este valor como parámetro para calcular algunas dimensiones de los componentes de la turbina (álabe y rodete), (Agar \& Rasi 2008). Otras dimensiones, como por ejemplo el diámetro del eje de la turbina y el espesor del rodete de la turbina, son calculadas con base a las características del material y el análisis de la integridad estructural usando un software de elementos finitos.

El Grupo de Energía Alternativa de la Universidad de Antioquia, usando la metodología mostrada 
en la figura 1, con el apoyo del Centro de Investigación e Innovación en Energía CIIEN y la misma Universidad de Antioquia, ha diseñado un microgenerador que genera $5 \mathrm{~kW}$ de energía

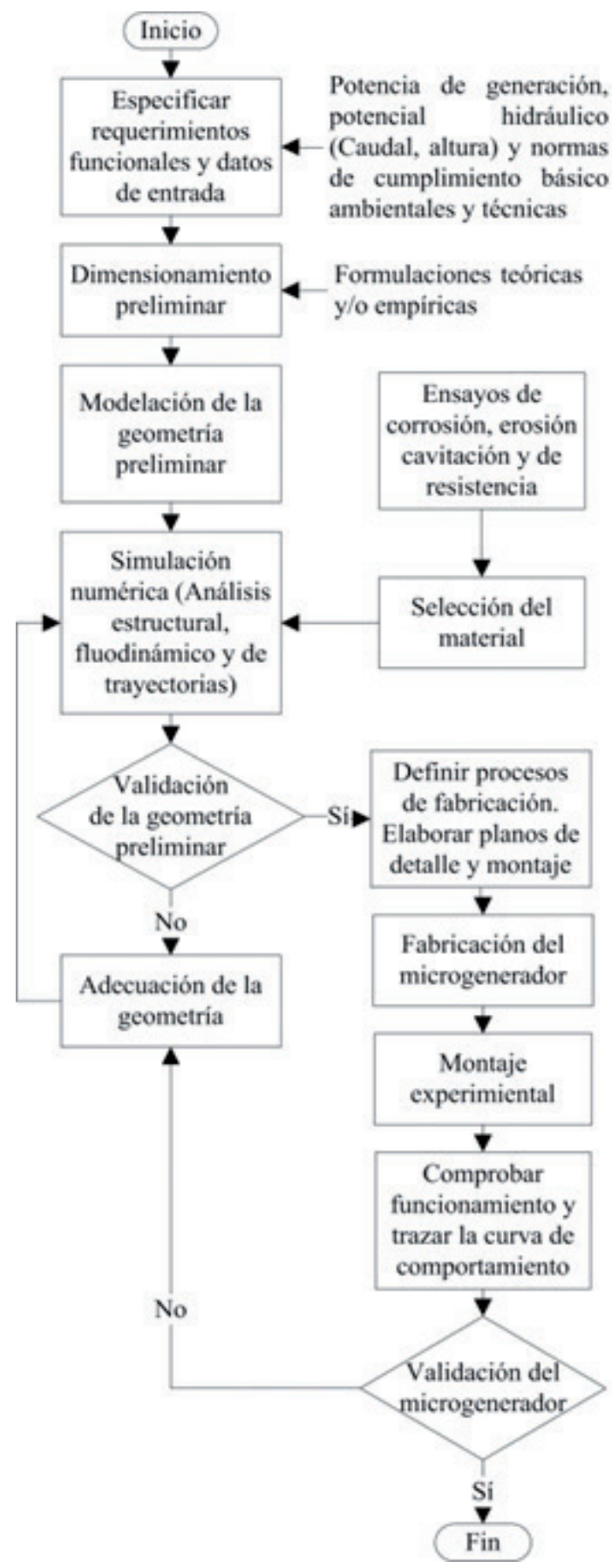

eléctrica para los siguientes parámetros de entrada: $0.03 \mathrm{~m}^{3} / \mathrm{s}$ caudal de diseño; velocidad de rotación de la turbina, $400 \mathrm{rpm}$; cabeza hidráulica, $30 \mathrm{~m}$; eficiencia de generación 51\%. Los

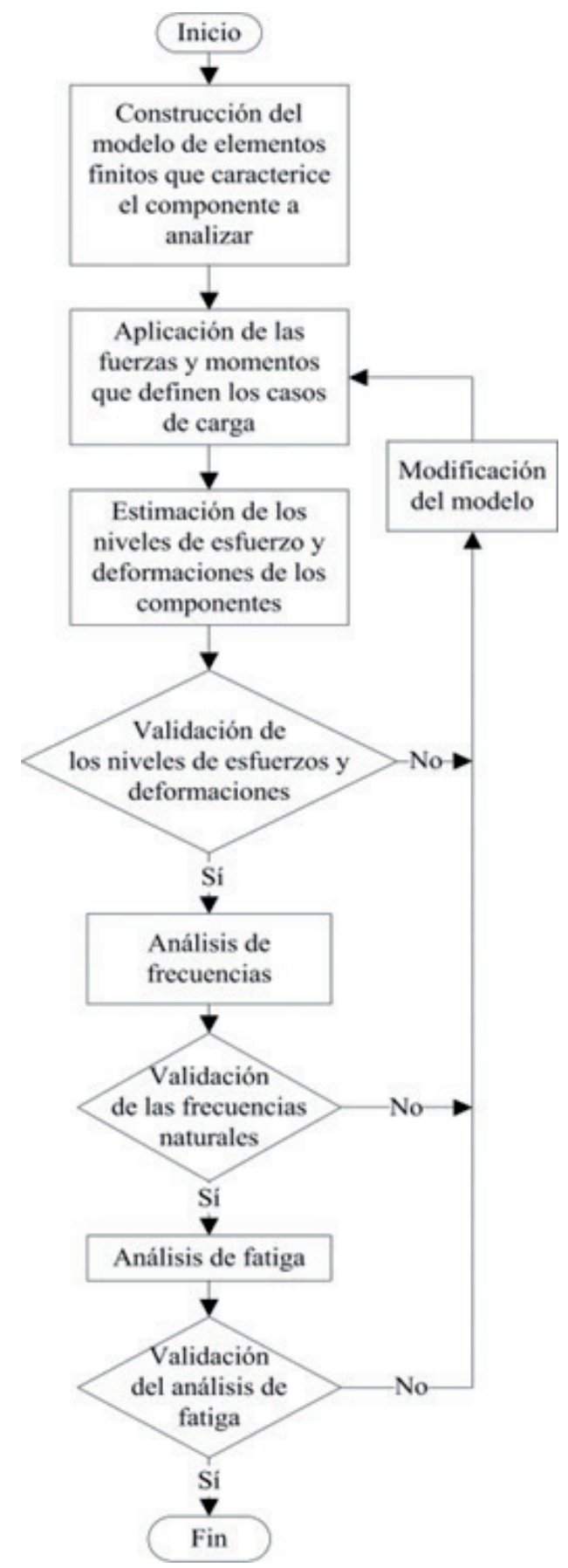

Figura 1. Flujo de información empleadas en el diseño de la turbina tipo Pelton. Lado izquierdo: Metodología para el diseño y fabricación del microgenerador. Lado derecho: Metodología para el análisis estructural de los componentes 
parámetros caudal de diseño y cabeza hidráulica fueron seleccionados por ser representativos de la geografía regional. La velocidad de rotación de la turbina se seleccionó teniendo en cuenta la velocidad específica de las turbinas Pelton.

Mediante la revisión bibliográfica se obtuvieron una serie de relaciones teóricas y empíricas que relacionan entre sí el diámetro del chorro con los parámetros geométricos del inyector, álabe y rodete. Estas relaciones permitieron modelar preliminarmente los componentes principales del microgenerador utilizando el software CAD SolidWorks (ver figuras 2 y 3 ).

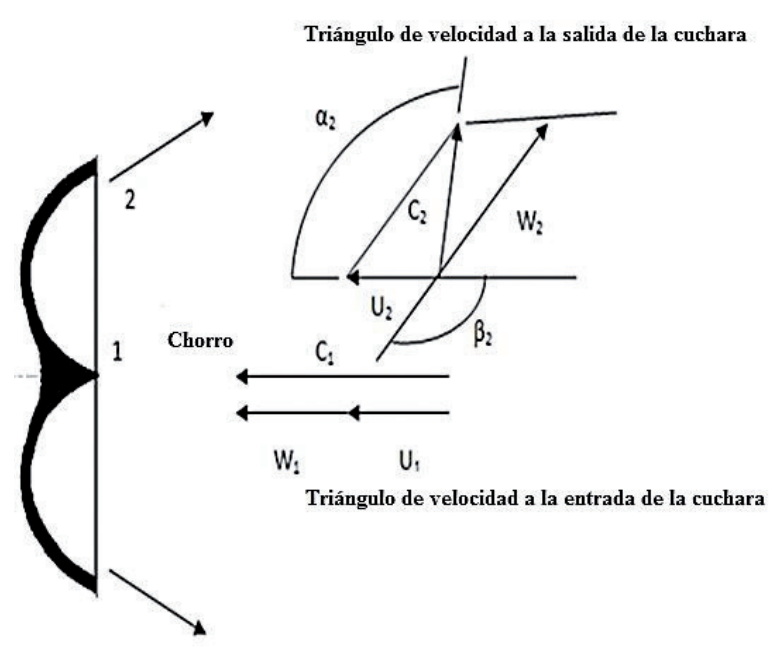

Figura 2. Triángulo de velocidades sobre el álabe.

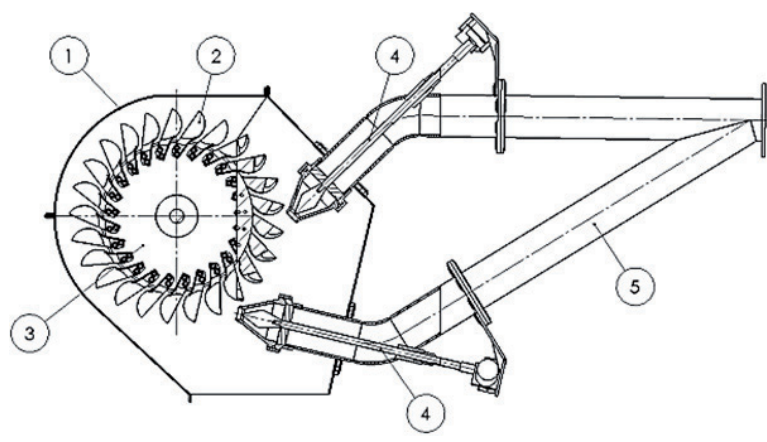

Figura 3. Componentes principales del microgenerador diseñado. 1) Carcasa, 2) Cangilones, 3) Disco circular, 4) Injector, 5) Tuberia de distribución.
Para transmitir la potencia del eje del microgenerador al generador es posible emplear un sistema de transmisión por correas y poleas que puede ser calculado con la potencia transmitida $(5 \mathrm{~kW})$, el factor de servicio y la velocidad de rotación de la turbina (400 rpm) y del generador; este último corresponde a un generador sincrónico de $1800 \mathrm{rpm}$.

\subsection{Análisis fluido-dinámico}

Se realizó un análisis de la turbina en el entorno del software CFD Flow Simulation (SolidWorks). El análisis contempla la modelización de flujo tridimensional turbulento confinado en el inyector y el flujo con superficie libre en los chorros, cangilones y carcasa. Los resultados del análisis permiten evaluar y modificar, eventualmente, los chorros de agua o validar el diseño de cangilones en lo que respecta a su geometría.

A partir de la geometría preliminar del microgenerador se define el dominio del fluido (volumen de control) sobre el cual se quiere tener información. Este dominio se discretiza o divide espacialmente en celdas para formar una malla. La distribución de velocidades y presiones del fluido son calculadas con la ayuda del software CFD.

A la entrada del volumen de control se impuso la presión dada por la cabeza hidráulica que aprovecha el microgenerador. A la salida, se impuso una presión igual a la presión atmosférica. Las velocidades sobre las paredes sólidas se asumen igual a cero excepto en las paredes de los álabes, el rodete y el eje, a las que se les impuso una condición de pared en movimiento (velocidad angular igual a $400 \mathrm{rpm}$ ). En este análisis se utilizó el modelo k- $\varepsilon$ para modelar la turbulencia, donde $\mathrm{k}$ es la energía cinética turbulenta y $\varepsilon$ su velocidad de disipación. En la figura 4 se muestra la distribución de velocidades en el plano medio del microgenerador.

Los resultados obtenidos de la solución de las ecuaciones se corresponden con el valor de las variables de campo en cada punto de la malla. En 

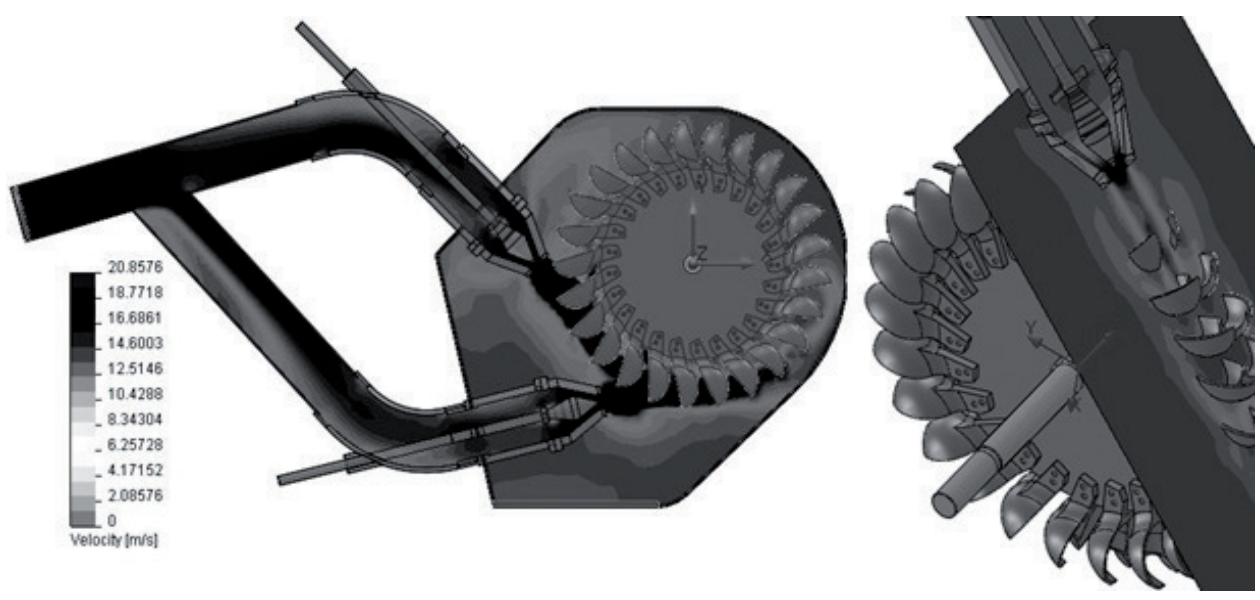

Figura 4. Análisis fluido-dinámico del microgenerador

general, existe una buena concordancia entre la simulación y los resultados analíticos calculados previamente en cuanto a la velocidad de salida del chorro, la fuerza sobre los cangilones y la potencia generada en el eje de la turbina. Para una cabeza de $30 \mathrm{~m}$, un caudal de $0.03 \mathrm{~m}^{3} / \mathrm{s}$, los resultados muestran una velocidad del flujo a la salida del chorro de $20.9 \mathrm{~m} / \mathrm{s}$ y una fuerza del chorro de 348.69 N. A partir de estos datos, además de la geometría y la velocidad de giro del rodete $(400$ rpm), se obtiene una potencia generada en el eje de la turbina por los dos inyectores de $5494.79 \mathrm{~W}$.

De los resultados del análisis, se concluyó que se dispone de la máxima energía cinética en cuando el agua incide tangencialmente sobre el rodete, empujando a los cangilones que lo forman, obteniéndose el trabajo mecánico deseado. Los chorros del fluido inciden sobre la arista diametral que divide a cada álabe en dos partes iguales, produciéndose una desviación simétrica en dirección axial, garantizando el equilibrio dinámico de la turbina Pelton en esa dirección. Las formas cóncavas de los cangilones hacen cambiar la dirección del chorro de agua por los bordes laterales, sin ninguna incidencia posterior sobre los cangilones sucesivos. De este modo, el chorro de agua transmite su energía cinética al rodete, donde queda transformada instantáneamente en energía mecánica, Xiao et al., (2007)

\subsection{Análisis estructural}

En la figura 1 se muestra el diagrama de flujo de la metodología empleada para realizar el análisis estructural que tiene por objeto garantizar la integridad estructural de cada uno de los componentes del microgenerador. En esta etapa de la metodología se consideran las diferentes alternativas de producción para cada pieza, además de los sistemas adecuados para su ensamble, lubricación y hermeticidad.

Para el análisis de los componentes principales del microgenerador se emplea el software de elementos finitos SOLIDWORK SIMULATION. En el software se definen las condiciones de carga $y$ frontera a las que se encuentran sometidos y las propiedades del material usado en su fabricación. Se verifican con la ayuda del software las tensiones y deformaciones de los componentes; además, se determinan sus frecuencias naturales. El análisis estructural se centró principalmente en los álabes o cangilones, el rodete, el eje y en los inyectores.

Para la fabricación de los componentes de la microturbina se seleccionaron algunos materiales que exhibieran buenos resultados ante pruebas de resistencia a la corrosión, erosión y cavitación. En este caso, se seleccionó el acero inoxidable 316 para fabricar el disco del rodete y el eje de 
la turbina. Para los álabes, después de probar diversos materiales, se prefirió una aleación de bronce al níquel, debido a su límite elástico, su tenacidad y resistencia al desgaste superficial y a la corrosión, además de su fácil maquinado, Choi et al (2006).

A continuación, se detalla el análisis estructural del cangilón. Éste se diseña para resistir el empuje directo del chorro de agua; por lo tanto, con los parámetros de entrada se ha calculado la fuerza del chorro; ésta es aproximadamente de $326.72 \mathrm{~N}$, muy similar a la calculada con el software CFD. Se realizó un mallado del componente en el software $\mathrm{CAE}$, el tipo de análisis realizado fue no lineal, se fijaron los agujeros de la base y el material asignado al modelo fue el bronce al níquel.

En la figura 5.a se muestra la distribución de tensiones y desplazamiento sobre el cangilón. Como se puede apreciar en la escala de grises de la figura, el mayor valor de tensión es 15.33 MPa y al compararlo con el valor de la tensión permisible del material de $275 \mathrm{MPa}$, se puede concluir que el material resiste la condición de carga aplicada.

En la figura 5.b se muestra el resultado de los desplazamientos, el mayor valor corresponde a $0.01638 \mathrm{~mm}$; el cual es menor que la luz existente entre carcasa y álabe, lo que asegura que no habrá interferencia entre estos componentes.

Los cangilones se encuentran expuestos a desgaste, picado, golpes y grietas que son ocasionados por mecanismos de daño como erosión, cavitación, impacto, fatiga y corrosión-fatiga. El mecanismo que representa mayor riesgo para su operación es la fatiga, conllevando a la iniciación y propagación de grietas que pueden afectar la integridad estructural del cangilón. Además, debido a la pérdida de material en el perfil de los cangilones, cambiarán las condiciones originales de diseño y esto puede causar vibraciones suficientemente serias cuando es muy avanzada, poniendo en peligro el funcionamiento mecánico del microgenerador, provocando variación del comportamiento hidráulico, Ferreño et al., (2011).
Dada la importancia del fenómeno de la fatiga en el cangilón también se realizó un estudio de este fenómeno en el software CAE. La información de diseño, fabricación y operación permitieron calcular los esfuerzos actuantes en el cangilón, siendo estimadas las hipótesis de carga a ser consideradas en el análisis de fatiga a partir de estos valores. Se asumen como ciclos o impactos, en un cangilón, el número de veces que un cangilón es atacado por un chorro. Esto es comparable a cargar y descargar sucesivamente el material, y es lo que influye en forma directa en el fenómeno de fatiga. Se ha estimado el número de ciclos de carga considerando un tiempo de operación del microgenerador de 8 horas diarias durante 3 años; además, se tiene en cuenta la influencia del número de arranques y paradas del microgenerador. De este análisis se ha estimado el porcentaje de daño del álabe respecto a la vida infinita. El análisis arrojó que todo el canjilón presenta el mismo resultado, por la cual se espera que la secuencia de eventos de fatiga consuma el $0.21 \%$ de la vida del álabe, lo cual es razonable y seguro.

Análisis similares al del cangilón han sido realizados para los demás componentes principales del microgenerador. Por ejemplo, además de los esfuerzos y deformaciones (Ver figura 5.c), en el eje se calculó la velocidad critica de flexión $\left(\mathrm{N}_{\mathrm{cr}}=5639.24 \mathrm{rpm}\right)$ y con la ayuda del software $\mathrm{CAE}$ se determinaron las frecuencias naturales del eje para garantizar una operación libre de vibraciones (Mataix, 1982).

\subsection{Fabricación del microgenerador}

Una vez validado el perfil hidráulico y garantizada la integridad estructural de los componentes, se procedió a la generación de los planos de fabricación y ensamble del microgenerador. Se emplearon diferentes procesos de fabricación como: maquinado convencional, control numérico y fundición, entre otros, dependiendo del componente a fabricar. Por ejemplo, para la fabricación de los álabes se exploraron tres alternativas: fundición a la cera perdida (investimento), fundición en moldes de yeso 


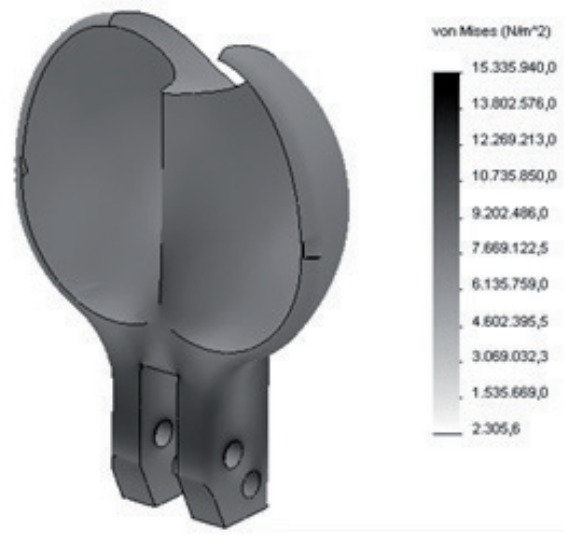

a)

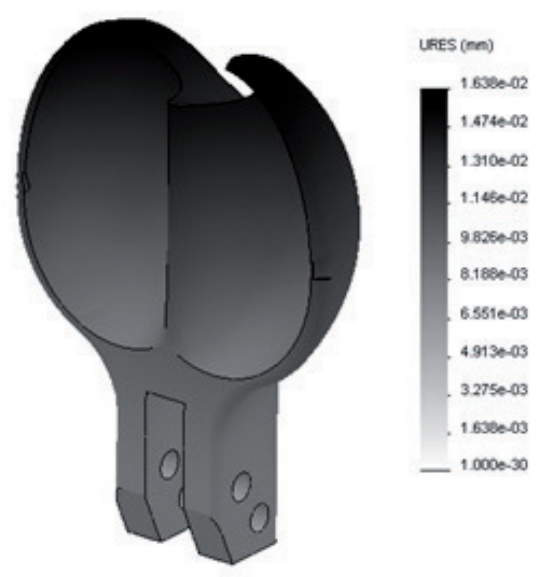

b)

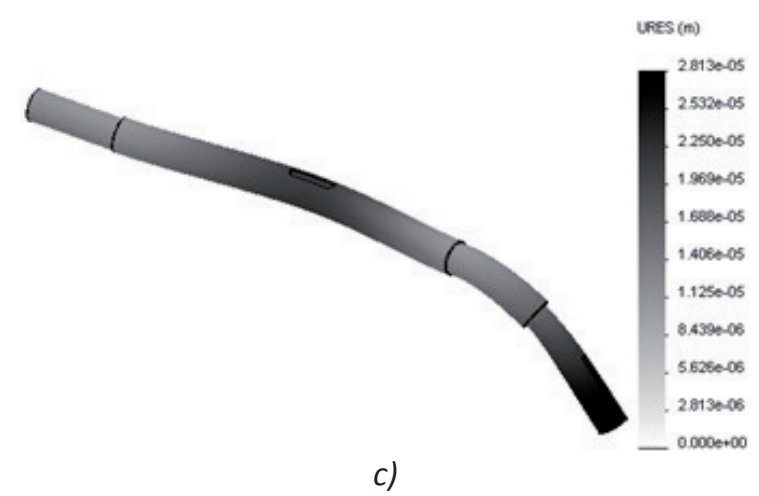

Figura 5. Algunos resultados del análisis estructural de los componentes de la turbina Pelton. a) Esfuerzos de Von mises del álabe, b) Desplazamientos del álabe, c) Deformación amplificada del eje

y fundición en arena (molde de caja fría). La selección de la mejor alternativa se hizo teniendo en cuenta la facilidad de moldeo, rapidez de moldeo, costos, versatilidad e imperfecciones finales en las piezas, Dragu et al., (2001).

Cualquiera de los procesos de fundición elegidos requiere de un modelo o prototipo para obtener el molde; para tal fin se empleó una impresora rápida de prototipos en $3 \mathrm{D}$ de marca ZPrinter con la cual se obtuvo el modelo del álabe diseñado.

Con el fin de simular el llenado y solidificación del álabe durante el proceso de fundición, se empleó el software SOLID CAST. Con esto se detecta prematuramente los problemas que se pueden presentar como rechupe, punto caliente o desgarre durante el proceso de fundición y permite realizar las correcciones correspondientes. Los datos de entrada del software son el material del molde, el material fundido y el modelo de la pieza a fundir, incluyendo las dimensiones del vertedero. Se realizaron diferentes simulaciones variando las dimensiones del vertedero y se obtuvo el tiempo y la cantidad de llenado, así como el tiempo de solidificación del álabe. El proceso de fundición en molde de yeso y en cera perdida presentó graves problemas de porosidad y rechupe en los álabes debido a bajos tiempos de calcinación del molde y erosión al molde refractario por el metal debido a la alta temperatura de calcinación. En la figura 6.a se presentan algunas de las piezas obtenidas donde se evidencian los problemas encontrados.

El proceso de fundición con molde de caja fría exhibió el mejor resultado. En este proceso, el prototipo se utiliza como modelo de fundición utilizando una placa falsa en yeso. La formaleta se emplea para los moldes. Se utilizó resina fenólica "Resol" de curado en $\mathrm{CO}_{2}$. La figura 6.b muestra los moldes superior e inferior utilizados en el proceso y se presentan algunos de los álabes fabricados.

\subsection{Montaje}

Con base en los planos realizados en la etapa de diseño se procedió al montaje de los diferentes componentes del microgenerador. En la figura 7.a se presenta la disposición del ensamble de los componentes del microgenerador y en las figuras 


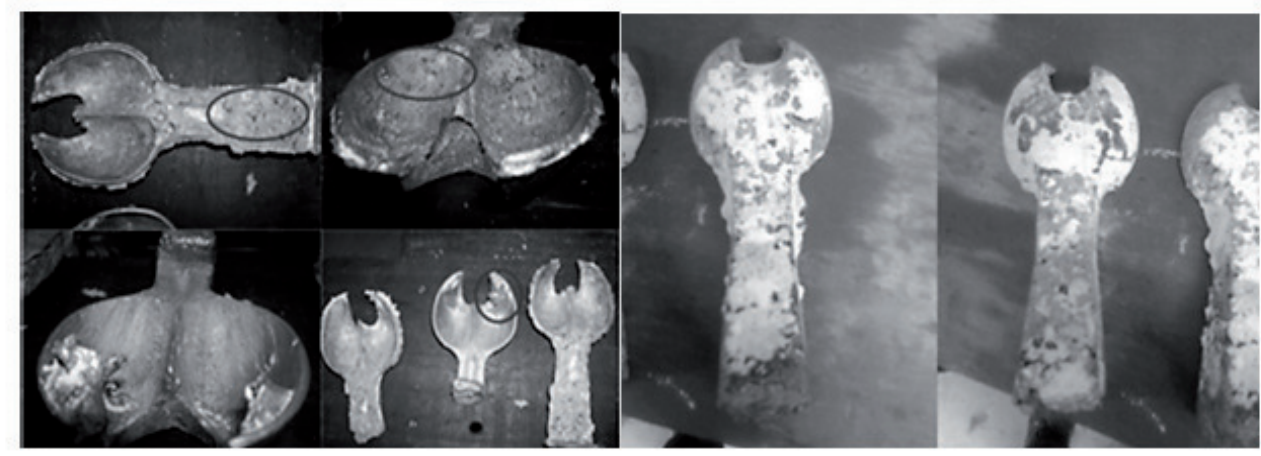

a.

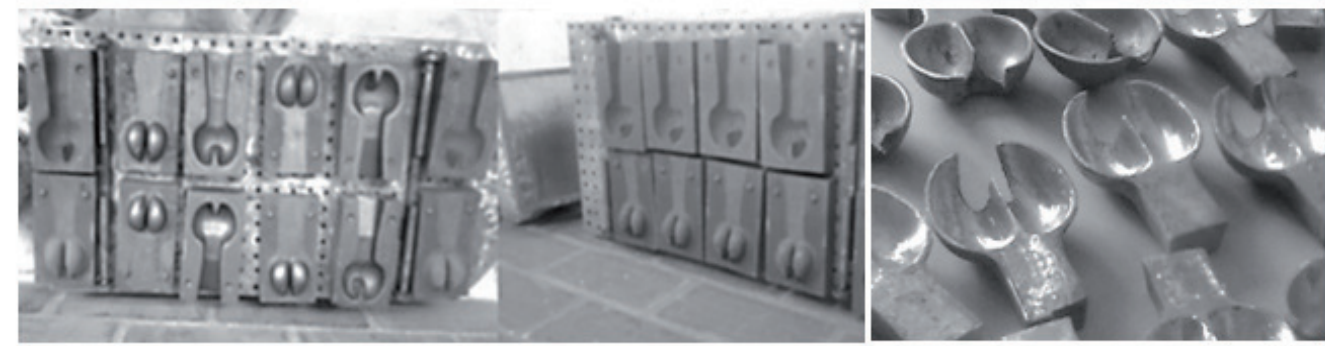

b.

Figura 6. Proceso de fabricación de los álabes de la turbina Pelton.

a) Piezas obtenidas con fundición a la cera perdida y con molde de yeso. b) Moldes de caja fría y piezas obtenidas.

7.b, 7.c y 7.d se presentan algunas fotografías del sistema ensamblado.

\subsection{Pruebas y resultados}

Las pruebas de funcionamiento del microgenerador hidráulico fueron realizadas en el laboratorio del Grupo de Energía Alternativa de la Universidad de Antioquia. Este laboratorio cuenta con un banco de caracterización de microgeneradores en donde se cuantificaron los distintos parámetros que intervienen en el funcionamiento del sistema a diferentes condiciones de operación. Todo esto se logra por medio de distintos sensores y de un sistema SCADA basado en PLC.

Debido a algunas limitantes en el laboratorio, en cuanto a la cantidad de caudal suministrado al microgenerador, sólo fue posible operarlo con un inyector y un caudal inferior a $0.014 \mathrm{~m}^{3} / \mathrm{s}$. Las pruebas consistían en operarlo bajo diferentes condiciones tanto de potencia hidráulica como eléctrica, donde se obtuvieron eficiencias entre el
$42 \%$ para generación a baja potencia eléctrica y el $50 \%$ para generación cercana $5 \mathrm{~kW}$, potencia de diseño (Ver figura 8.a).

En la figura 8.b se observa que el microgenerador opera en el rango de eficiencia especificado cuando la velocidad de la turbina está alrededor de $400 \mathrm{rpm}$, (velocidad de diseño), lo que garantiza que la eficiencia de diseño se mantenga cuando el microgenerador opere bajo condiciones nominales de generación; es decir, con una amplitud y frecuencia eléctrica de la onda de voltaje generada alrededor de $60 \mathrm{~Hz}$ y $110 \mathrm{VAC}$, respectivamente.

Ya que la potencia hidráulica es proporcional a la cabeza hidráulica y al caudal aplicado, en las figuras 8.c y 8.d se observa que el microgenerador presenta una baja eficiencia cuando se opera con una potencia hidráulica baja y una alta eficiencia cuando se aplica una potencia hidráulica alta, cercana a la potencia hidráulica de diseño. 


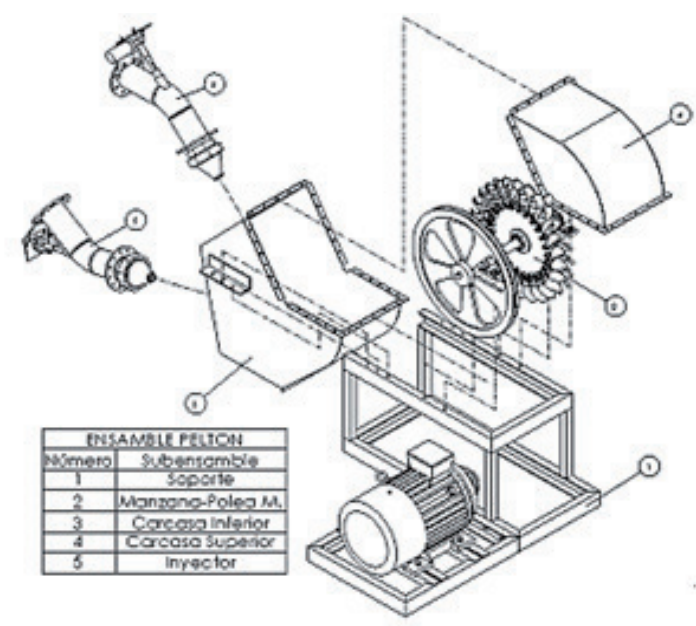

a)

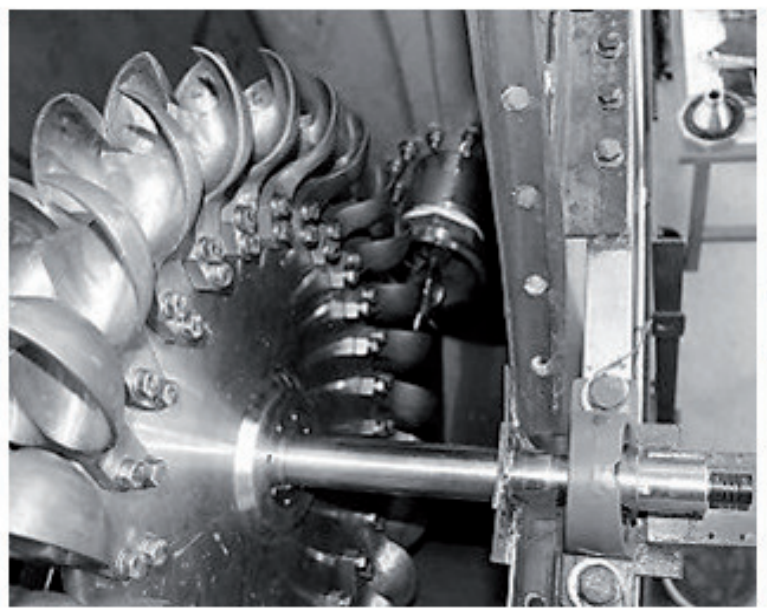

c)

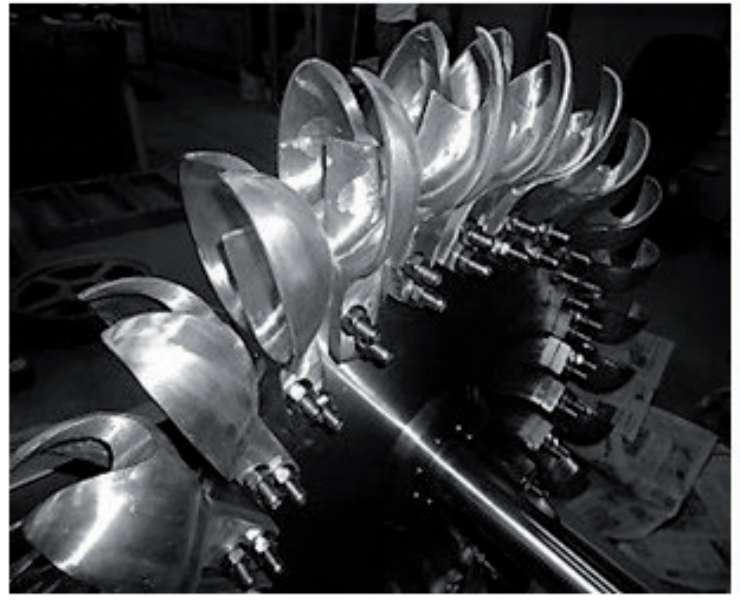

b)

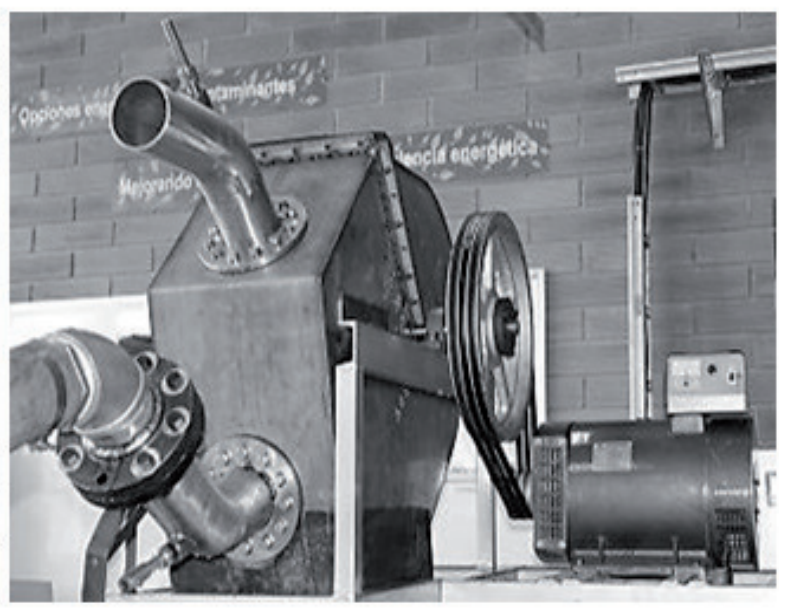

d)

Figura 7. (a) Layout microgenerador b) fotografía álabe microgenerador construido, c) fotografía álabe e inyector microgenerador construido), d) fotografía microgenerador construido

Además, en la figura 8.c se observa que la eficiencia para la cabeza hidráulica de diseño (30 m) está alrededor de $48 \%$, y que la eficiencia máxima se presenta para una cabeza mayor, debido a que al aumentar la cabeza hidráulica se compensa el bajo caudal aplicado. Así, la máxima eficiencia obtenida se logra alrededor de la potencia hidráulica de diseño, Gilli et al., (1998).

Aunque las condiciones a las que fue sometido el microgenerador no son las de diseño y sólo se utilizó un inyector, los resultados de estas pruebas dan garantías sobre una eficiencia global de generación cercana, e inclusive superior, al valor sugerido en la etapa de diseño.

\section{Conclusiones}

Se propuso una metodología para diseñar, construir y caracterizar un microgenerador hidráulico, a partir de una base teórico-experimental. Se emplearon herramientas computacionales para apoyar la etapa de diseño y fabricación de los componentes, con lo cual se redujeron los costos 


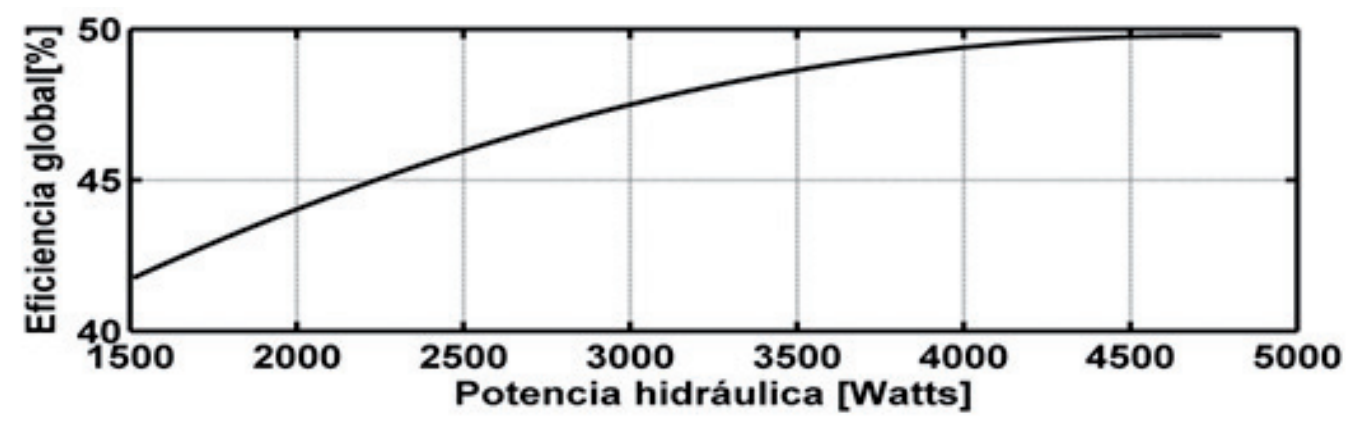

a)

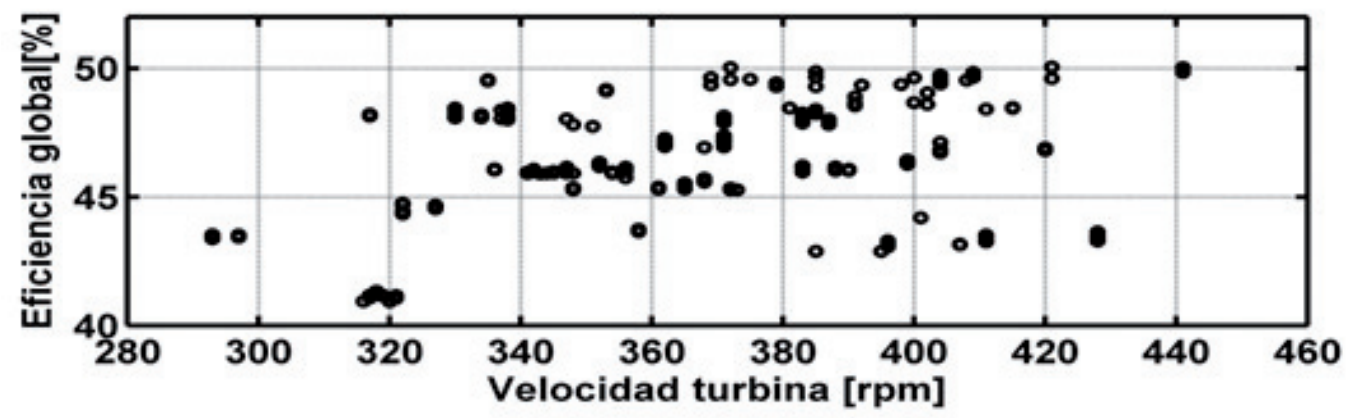

b)

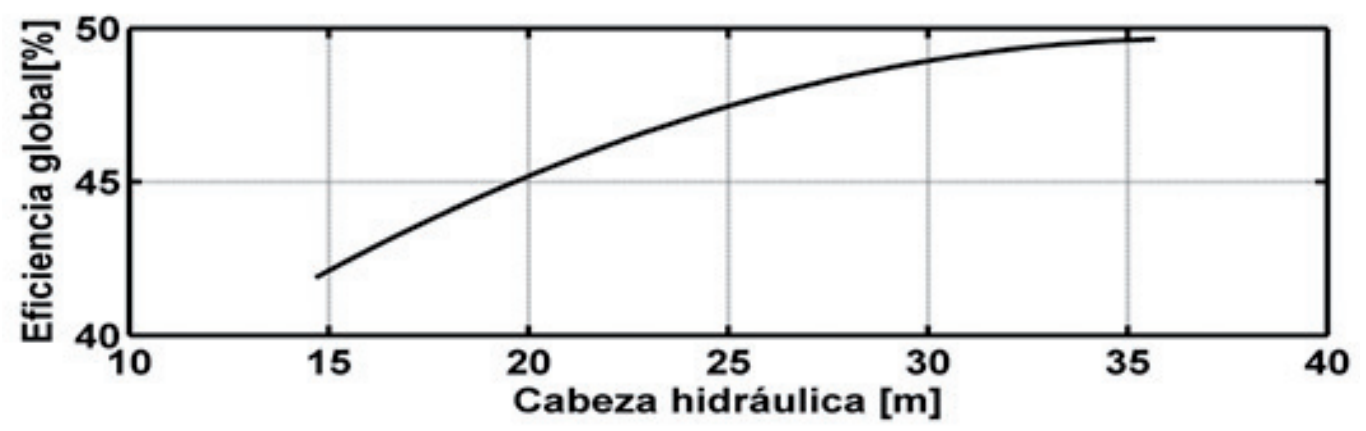

c)

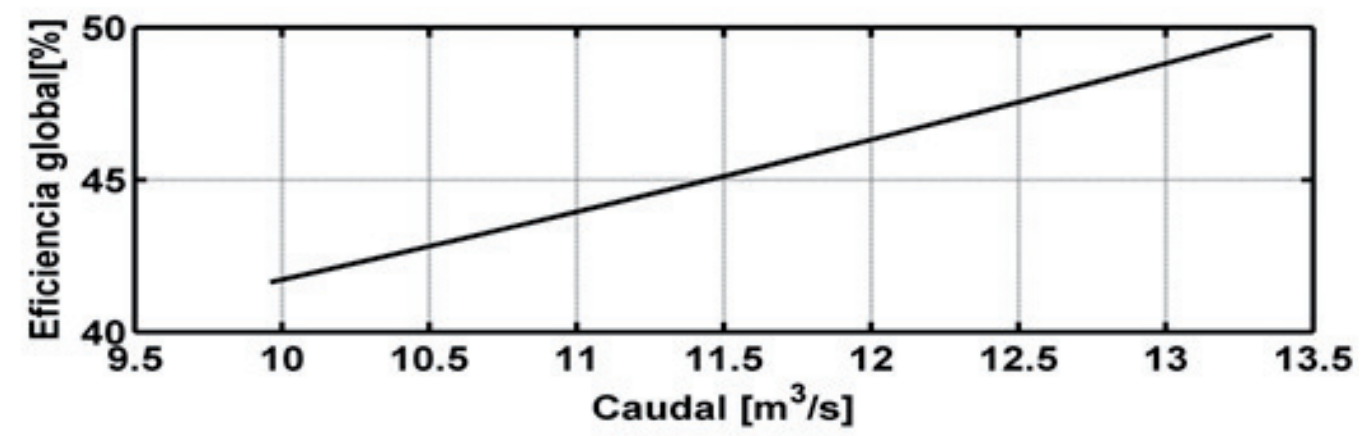

d)

Figura 8. Eficiencia global [\%] de las pruebas de banco realizadas a la turbina Pelton a diferentes condiciones. a) Potencia hidráulica [Watts], b) Velocidad de la turbina [rpm], c) Cabeza hidráulica [m], d) Caudal [m³ $/ \mathrm{s}]$. 
de desarrollo y fue posible experimentar, mediante simulaciones el efecto que generaron algunos cambios en la geometría de los componentes del microgenerador.

El empleo de esta metodología permitió obtener un microgenerador hidráulico que se infiere un funcionamiento eficiente en las condiciones hidráulicas para las cuales fue diseñado ya que a las condiciones que fue evaluada en el laboratorio de Energía Alternativa de la Universidad de Antioquia tuvo un comportamiento adecuado. Además, mediante estas pruebas de banco se pudo validar el diseño del microgenerador $\mathrm{y}$ algunos resultados obtenidos previamente en las simulaciones computacionales.

Se presentaron diferentes procesos de fabricación para elaborar álabes de turbina Pelton. Los resultados obtenidos permitieron seleccionar el proceso de fundición con molde de caja fría como idóneo para fabricar este tipo de álabe.

La metodología propuesta puede ser empleada para el diseño y construcción de microgeneradores hidráulicos, para diferentes capacidades de generación y que utilicen otros tipos de turbinas.

\section{Referencias bibliográficas}

Agar, D. \& Rasi, M. (2008). On the use of a laboratory-scale Pelton wheel water turbine in renewable energy education. Renewable Energy, $33,7,1517-1522$.

Choi, Y., Kurokawa, J., Nakajima, N., \& Phommachanh, D. (2006). Development of a Positive Displacement Micro-Hydro Turbine. JSME International Journal Series B, 49, 2, p. 482-489.
Dragu, C., Sels, \& T., Belmas, R. (2001). Small Hydro Power State of The Art and Applications. International conference Power Generation and Sustainable Development (AIM), Liege, Belgium, 265-270.

Ferreño, D., Álvarez, J.A., Ruiz, E., Méndez, D., Rodríguez, L., \& Hernández, D. (2011). Failure analysis of a Pelton turbine manufactured in soft martensitic stainless steel casting. Engineering Failure Analysis, 18, 1, 2011, 256-270.

Gilli, J., Massaro,F., \& Pagnola, M.(1998).Reporte técnico $n^{\circ} 1$ - abril 1998. Propuesta de diseño - banco de pruebas experimentales. Proyecto: microturbinas hidráulicas CFT, caracterización. Grupo de microturbinas hidráulicas Universidad Nacional de Lomas de Zamora - Facultad de Ingeniería. Buenos Aires -Argentina.

ITDG Soluciones Prácticas (n.d). Ficha Técnica $N^{\circ} 25$ Turbinas Pelton. Consultado el 30 de octubre, página web de la organización Soluciones Prácticas ITDG, Lima-Perú. http:// www.solucionespracticas.org.pe/fichastecnicas/ pdf/FichaTecnica25-turbinas\%20pelton.pdf

Mataix, C. (1982). Mecánica de Fluidos y Máquinas hidráulicas. 2da ed. Oxford University Press, 1982.

OLADE, Organización Latinoamericana de Energía (1988). Apuntes para un manual de diseño, estandarización y fabricación de equipos para pequeñas centrales hidroeléctricas. Universidad Nacional de la Plata.

Xiao, Y., Han, F., Zhou, J., \& Kubota, T. (2007). Numerical prediction of dynamic performance of Pleton turbine. Journal of Hydrodynamics, Ser. B, 19, 3, 356-364. 